\title{
Collaboration on Interactive Ceilings
}

\author{
Alexander Bazo, Raphael Wimmer, Markus Heckner, Christian Wolff \\ Media Informatics Group, University of Regensburg
}

\begin{abstract}
In this paper we discuss how interactive ceilings may improve productivity and collaboration in office environments. The ceiling of an office offers an unobtrusive und generally unobstructed display and input area which is accessible to all persons in the room. Therefore, enhancing ceilings with input and display capabilities allows for a range of new single- and multi-user applications such as status indicators, notifications, in-house navigation and collaborative work areas. However, ergonomic constraints limit the application space. To investigate such constraints we have built a working prototype of an interactive ceiling. Currently, we are conducting a study investigating which areas on the ceiling may be used for displaying notifications and content.
\end{abstract}

\section{Interactive Ceilings}

Interactive surfaces support collaboration between co-located and remote co-workers. They allow multiple collaborators to simultaneously view and manipulate shared data. Beside personal displays and tablet devices, interactive tabletops and wall-mounted interactive whiteboards are commonly used. However, interactive tabletops and whiteboards suffer from several limitations that discourage quick, ad-hoc collaboration. All collaborators have to walk up to the table or wall to interact with them. Content on tabletops is only visible to users standing right next to the device. Content on wall-mounted displays gets occluded by other people or objects standing in the line-of-sight. Without proper software support, exchanging data between personal computing devices and collaborative interactive surfaces is cumbersome. Tabletops and walls offer only limited display space which often allows only a subset of all relevant data to be displayed at once. In our experience, these limitations make shared interactive surfaces so tedious to use that people rarely use them for ad-hoc collaboration and sharing of data.

Most office spaces have a ceiling. It differs from other surfaces in the room in several characteristics: In general, ceilings offer large, uninterrupted flat white areas. Unlike walls, they are rarely occluded or interrupted by doors, windows and furniture and are visible from 
any position in the room. Lighting fixtures in office environments are rather unobtrusive and often embedded into the ceiling.

Each location on the floor may be mapped to a location on the ceiling. Thereby, information displayed on the ceiling may be associated with persons or objects below it. Such ceilings may be easily turned into large interactive displays by installing computer screens or projecting screen contents from below. These may be viewed and used simultaneously by multiple users. Unlike interactive whiteboards or tabletop systems an interactive ceiling does not imply preferred positions for users and can not be visual blocked by other users. Therefore, users may interact with and on the ceiling from their desks. This facilitates ad-hoc interaction and allows for better integrating personal computing devices with a shared interactive surface. In typical settings for large office spaces with cubicle desks providing some kind of privacy in the large room, interaction via the ceiling may still be possible.

We define interactive ceilings as display surfaces which cover ceilings partly or completely, allow visualization of digital content and offer input techniques for user interaction. For investigating the potential of interactive ceilings we have built a prototypical setup of such a surface. We have already discussed properties and some general usage scenarios (Wimmer et al., 2013). In this paper we present preliminary findings of a user study on visual perception of content on the ceiling and discuss its potential for collaborative use.

\section{Related Work}

Ceilings have long been a topic of research in architecture. However, few researchers have investigated the potential of interactive ceilings so far. Pieper and Kobsa (1999) present a ceiling-mounted display for bed-ridden users. Martin Tomitsch explores basic properties of interactive ceilings and has implemented several ambient information displays on a ceiling (Tomitsch, 2007,2008; Tomitsch \& Greching, 2007). Meagher (2010) discusses architectural aspects of ceilings as display surfaces. The collaborative use of interactive ceilings has not been investigated so far.

\section{Perception and Ergonomics}

Whereas wall displays and tabletops require users to look straight ahead or slightly downwards, interactive ceilings require users to look up. Depending on the location of content on the ceiling, users have to lift their gaze slightly or significantly. Obviously, looking upwards is rather tiring and may even hurt after some time. Therefore, a crucial objective of first ceiling interaction studies must be the users' perceptual and ergonomic constraints. Exploring these allows us to determine which areas of the ceiling are visible without lifting the head, and which areas are comfortable to look at for a longer time.

For this purpose we have designed and are currently conducting a study to identify a user's field of view depending on a) whether the user is sitting or standing, and b) how much the 
user turns their head upwards. Users stand or sit under the interactive ceiling, focusing fixed markers on the floor, wall and ceiling. Each marker represents a specific inclination of the user's line of sight. Those inclinations reflect different typical working situations as looking into a computer screen (slightly downwards), reading a book (downwards) or looking directly at the ceiling (heavily upwards).

While the user stares at the marker, a high-contrast target moves slowly into their field of view form different directions. Once the user recognizes it, he presses a button. This allows us to determine the area on the ceiling where one might be able to perceive notifications. Each target also has an embedded semi-random letter (letters are randomly chosen from the following set of symmetrically shaped letters ' $O, X, E, H, I, S$ ' to minimize misinterpretation by orientation) in it, representing notification content to be read. While the target is in the peripheral field of view, the user is not able to identify the letter. Once the user can read the letter, they press the button again. This allows us to determine the area on the ceiling where one might be able to read text or watch other content.

In our study, we have conducted two rounds with 2 (sitting/standing) x 6 (marker positions) $\mathrm{x}$ 4 (repetitions) $=48$ trials each. In the first round (see Fig. 1 left), users were asked to stare at the marker for the whole experiment. In this case, the letter within the target became only visible once the target moved into the user's central field of view. In the second round (see Fig. 1 right), users were allowed to focus on the target once it entered their peripheral field of view. This allows us to analyze both minimally and maximally available area for textual content on the ceiling.

Based on preliminary findings from four participants we have reason to believe that ceiling interaction is useful both for notifications and more complex use cases with higher attention needs. Realistic user poses (reading, desktop computer use) still allow users to notice visual stimuli on the ceiling within their peripheral field of view. Needless to say, facing the ceiling directly increases the chance to identify the character correctly. Users report that even though they are able to identify the targets and characters from lower angles, higher neck angles allow faster fixation of the targets. Furthermore all users report that they would use an interactive ceiling as a notification area or for other tasks that require occasionally looking directly at the ceiling (see Figure 1).
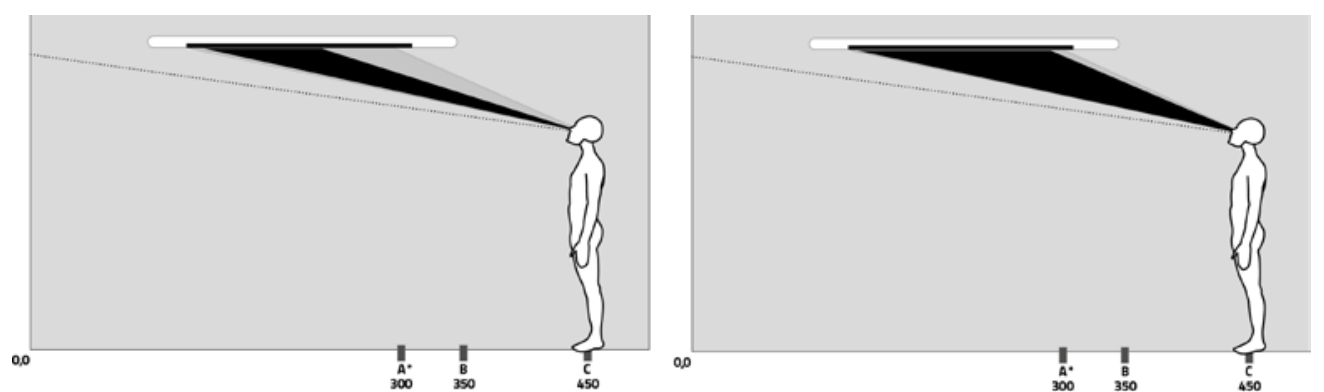

Figure 1: Field of view as determined in our study: Fixed eye gaze position (left), Non-fixed eye gaze position (right). Dark areas show users'central field of view. 


\section{Applications}

Based on the characteristics outlined above, we see three major use cases for interactive ceilings: (1) Interactive ceilings can be used to display ambient or highly visible notifications. (2) Interactive ceilings allow displaying content that is spatially connected to objects, waypoints or persons below it. (3) Interactive ceilings improve collaborative work by offering an open, rarely occluded interactive surface (see Figure 2).

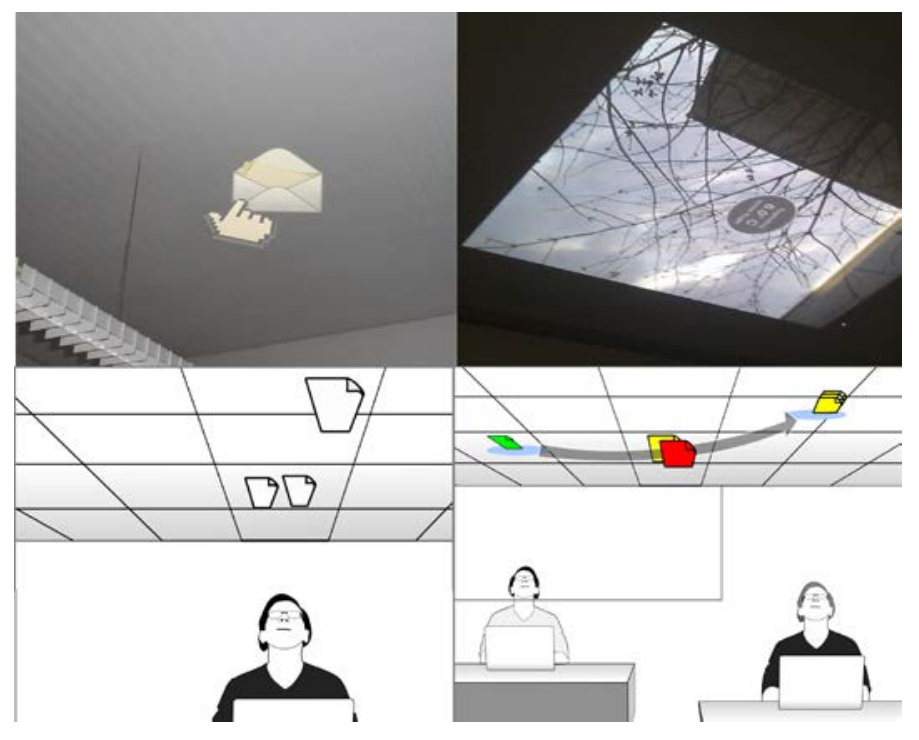

Figure 2: Applications (clockwise): Email notification, virtual rooflight with weather information, personal clipboard, document sharing

Notifications of incoming messages or due appointments could be displayed on the ceiling. As users notice visual stimuli in their peripheral field of view, an interactive ceiling may be used as an additional unobtrusive area for such information while the primary work area (e.g. a desktop computer's screen) remains available for main tasks. This can be combined with clipboard functions allowing users to temporally stash and share content out of view on the ceiling.

One important difference between ceilings and other interactive surfaces in a room is their direct mapping to the room's floor layout. This relation may be used to augment withinbuilding navigation by displaying directions and waypoints on the ceiling. External context information - such as the current weather outside - may be unobtrusively presented in a virtual window or roof-light with live visual feeds. 
Furthermore, the personal workspace of each user can be mapped to the ceiling area directly above. Users may share digital information with co-located co-workers by 'pushing' them across the ceiling towards the recipient's workspace. Status information about objects and persons (e.g. "do not disturb") may be presented above them on the ceiling.

\section{Collaboration Support: An Example Scenario}

Several characteristics of interactive ceiling may address collaboration. The large-scaled, always present display space offers a shared workspace allowing natural user interfaces for multi user workflows. Digital artifacts as files, messages or task representations may be transferred via the ceiling from one user to an other offering a quick exchange possibility. Users may not have to stand up from their desk for simultaneously using a shared workspace.

Spatial reference between floor and ceiling supports teamwork. Co-located persons may utilize the ceiling area above them as an ad-hoc shared display as it is easily sub dividable for multiple groups.

While implementing such scenarios we have to be aware of obvious limitations. For ergonomic reasons ("gorilla neck") ceilings should not be used continuously for long periods. As pointing may be inaccurate direct interaction should be limited or realized by other means. In addition the user interface itself may be hard to implement as the users share no common orientation or viewing angle while facing the ceiling.

The following "a day in the life of"-scenario for fictitious office worker Mary demonstrates the possibilities of ceiling interaction while avoiding mentioned limitations. Typical characteristics of interactive ceilings are mentioned in italics:

Mary enters the office. At a glance she sees the status of the whole team (status messages over the team members, spatial reference): Peter has 20 support tickets hovering over him, Max is busy and does not want to be disturbed and Michelle and Tom are obviously working together on a presentation - they are using a shared workspace on the ceiling to exchange illustrations.

Mary sits down at her desk and browses through her incoming messages. One is outside her area of expertise. As she glances to the ceiling, she notices that Tom has already a pile of messages waiting for him, while Jerry has only two messages (user information with spatial reference). Therefore, Mary forwards the message to Jerry. She drags it upwards to the ceiling and slides it towards Jerry's desk by pointing at the message and conducting a quick pushing gesture (physical workflows).

Mary notices Tamara joining Michelle and Tom: When she moves towards them, the shared area on the ceiling expands and Tamara's files are added to the stack of files shown on the ceiling (ad-hoc displays for co-located work). Some time later the three leave for coffee. Status indicators above their desks show everyone in the room that they will be back in a few minutes. 
Later on, Jim, a customer, arrives to talk to Mary about a recently started project. It is his first visit in this open-plan office and he does not know where Mary's cubicle is. He opens her contact information on his smartphone and the ceiling briefly shows navigational information to guide him towards Mary (spatial reference).

Leaving the office in the evening, Mary looks up at the ceiling, through the virtual roof light above the door (see Fig. 2, upper right). The live feed from a camera installed on the roof of the building shows her that it is raining outside. She goes back to her desk and fetches her umbrella.

\section{Outlook}

We are currently conducting the aforementioned study and plan to present results soon. A future study will investigate constraints for interaction on the ceiling. Following this, we plan to implement and analyze several novel interaction techniques for ceilings.

\section{References}

Meagher, M. Dynamic Ornament: The Design of Responsive Architectural Environments. PhD thesis,Ecole Polytechnique Federale de Lausanne, 2010.

Pieper, M., and Kobsa, A. Talking to the ceiling: an interface for bed-ridden manually impaired users. In CHI '99 Extended Abstracts on Human Factors in Computing Systems , CHI EA '99, ACM (New York, NY, USA, 1999), 9-10.

Tomitsch, M. Interactive Ceiling - Ambient Information Display for Architectural Environments .PhD thesis, Vienna University of Technology, 2008.

Tomitsch, M., and Grechenig, T. Reaching for the ceiling: Exploring modes of interaction. In Adjunct Proceedings of the International Conference on Ubiquitous Computing (Ubicomp07) (2007).

Tomitsch, M., Grechenig, T., and Mayrhofer, S. Mobility and emotional distance: exploring the ceiling as an ambient display to provide remote awareness. In Intelligent Environments, 2007. IE 07. 3rd IET International Conference on (Sept. 2007), $164-167$.

Wimmer R., Bazo A., Heckner M., Wolff C. Ceiling Interaction: Properties,Usage Scenarios, and a Prototype. In Blended Interaction (Workshop) at ACM SIGCHI Conference on Human Factors in Computing Systems, Paris 2013. 